\title{
Pour ne pas en rester aux déclarations d'intention concernant le compagnonnage pendant l'externat
}

La plupart des programmes de formation médicale prégraduée sont organisés, de par le monde, selon une architecture dite postflexnerienne, du nom d'Abraham Flexner qui, au début du XX ièele, rédigea un rapport critique et des recommandations concernant l'enseignement de la médecine en Amérique du Nord ${ }^{1}$. A deux ou trois années de cursus préclinique consacrées à l'enseignement des sciences fondamentales, succèdent trois à quatre années de formation dédiées aux sciences cliniques. Au cour du cursus clinique, l'externat sappuie sur des fondements pédagogiques implicites qui semblent robustes. Examiné selon une perspective traditionnelle de la formation professionnelle, il bénéficie ainsi de la solide et consensuelle caution que lui confere sa référence au modèle du compagnonnage. Revisité à la lumière des acquis récents des sciences de l'apprentissage, il réunit aussi a priori toutes les conditions permettant de satisfaire les exigences énoncées en référence aux cadres conceptuels fournis par la théorie de l'apprentissage expérientiel ${ }^{2}$ ou par celle de l'apprentissage contextualise $e^{3}$. Enfin, l'externat offre les conditions d'une prise progressive de responsabilités en situation authentique de pratique; en cohérence avec des éclairages théoriques développés pour d'autres professions, comme celle proposée dans le modèle dit de la "participation périphérique légitime " "4, qui décrit comment un " apprenti " migre progressivement d'une situation périphérique vers une position de plus en plus centrale dans un milieu professionnel, il constitue également un cadre propice au développement du processus de socialisation du futur médecin et à son acquisition d'une "culture de pratique".

Pourtant, l'externat fait l'objet depuis quelque temps de nombreuses critiques qui en dénoncent les limites et les insuffisances, la situation étant même décrite comme préoccupante dans un éditorial récent ${ }^{5}$. Dans ce contexte, le travail de Serge Langevin et René Hivon, qui parait dans ce numéro ${ }^{6}$, apporte une somme d'informations précieuses pour améliorer la valeur formatrice de l'externat et mieux exploiter son extraordinaire potentiel. A partir d'une revue exhaustive et méthodique de la littérature scientifique récente consacrée à l'externat, ils dressent d'abord l'inventaire de tous les facteurs susceptibles d'en menacer l'efficience pédagogique. Ils en proposent une classification structurée autour de quatre dimensions: l'organisation curriculaire, qui concerne les questions sur la place de l'externat au sein de l'ensemble du programme de formation et sur l'articulation des différentes séquences de stage entre elles; l'évaluation et le monitoring, qui permettent d'analyser les difficultés concernant les pratiques d'évaluation des apprentissages en contexte de stage; la qualité didactique, qui autorise une analyse critique des diverses ressources d'enseignement et d'apprentissage offertes dans le cadre d'un stage; et enfin l'environnement clinique, qui permet d'examiner la pertinence pédagogique d'un stage en tant que contexte de formation. En s'appuyant sur le cadre conceptuel ainsi élaboré et en recourant à une démarche de recherche qualitative conduite dans leur milieu institutionnel, les auteurs valident ensuite la viabilité opérationnelle de cette grille de lecture auprès des membres de leur communauté d'enseignants cliniciens et sappuient sur elle pour élaborer des hypothèses de solution et identifier les lignes directrices d'une réforme de l'externat.

Malgré un constat qui peut paraître sévère, il n'est bien entendu pas question de "jeter le bébé avec l'eau du bain ". Aucun dispositif d'externat, dans nos différentes facultés, ne cumule à lui seul tous les inconvénients et tous les défauts identifiés par cette étude systématique. Lorsquils sont avérés, les différents écueils ne sont en outre pas tous concentrés sur chacun des stages, obligatoires ou optionnels, qui jalonnent le parcours d'un externe. Dans de nombreux services, beaucoup d'enseignants cliniciens sont préoccupés d'exploiter au maximum toutes les opportunités d'enseignement et d'apprentissage pertinentes dans l'optique de la construction de compétences et de fournir pour ce faire les meilleures ressources aux étudiants quils supervisent, en démontrant par exemple un engagement en profondeur en tant que modèles de rôle explicites ${ }^{7}$. Il est en revanche loyal de reconnaître que le dispositif de l'externat, conçu comme une simple séquence de stages permettant aux étudiants d'appliquer concrètement des apprentissages effectués ailleurs, dans le cadre d'un accompagnement bienveillant et généreux mais peu formalisé et inadéquatement évalué, ne répond plus complètement aux exigences pédagogiques et aux attentes sociales, telles qu'elles sont aujourd'hui formulées dans le cadre des recommandations publiées par les organismes nationaux d'accréditation, par exemple dans le cadre du projet CanMEDS au Canada ou Tomorrow's doctors au Royaume-Uni.

Une conception implicite du compagnonnage, réduite à l'idée que l'accumulation d'expériences, par le biais 


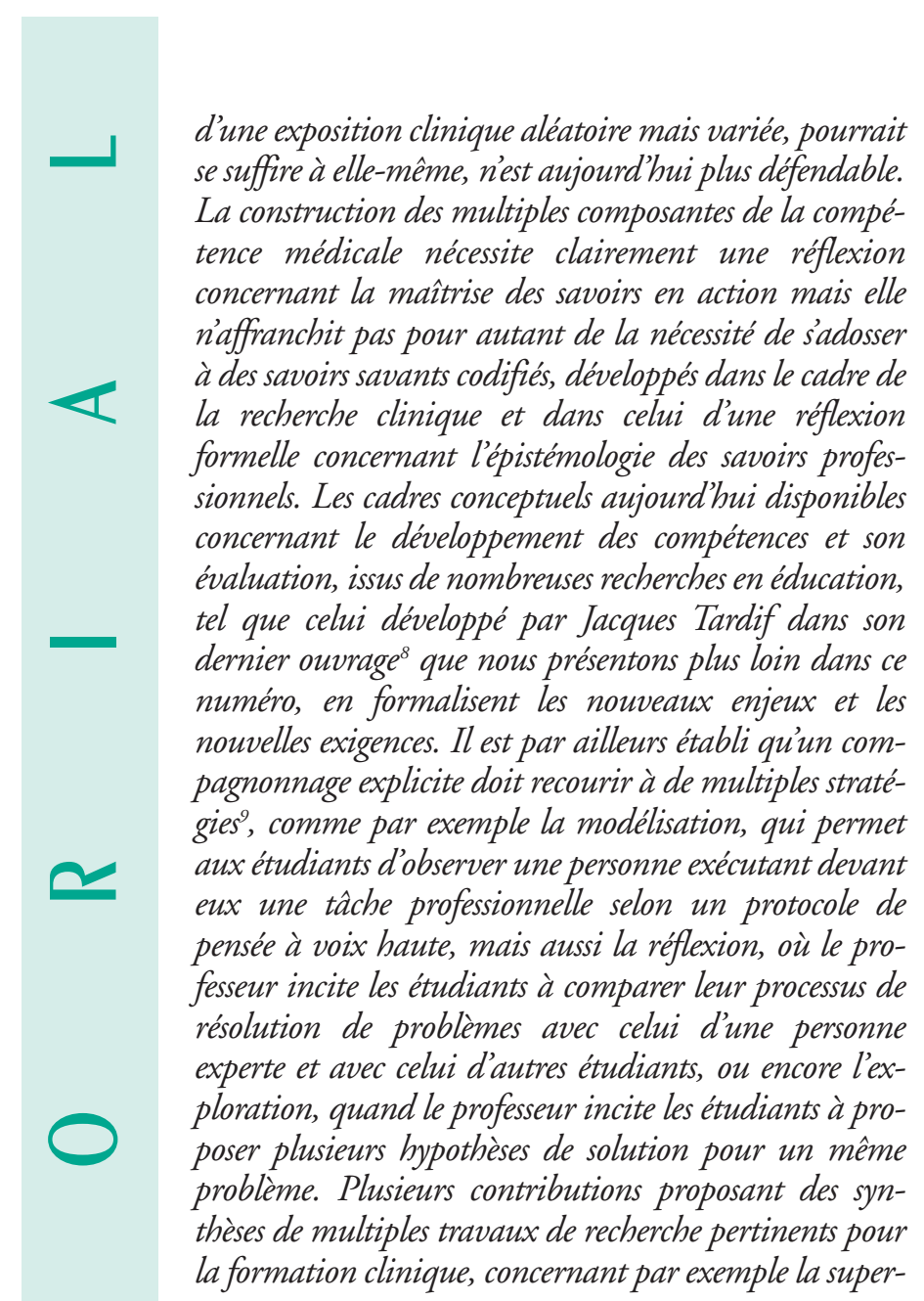

— Références

1. Flexner A. Medical Education in United sates and Canada. The Carnagie Foundation for the Advancement of Teaching 1910 ; 85.

2. Kolb D. Experiential learning. Experience as a source of learning and development. Englewood Cliffs (NJ):Prentice-Hall, 1984.

3. Brown JS, Collins A, Duguid P. Situated cognition and the culture of learning. Educational Researcher 1989;18:32-42.

4. Lave J, Wenger E. Situated Learning: Legitimate peripheral participation. Cambridge (UK): Cambridge University Press, 1991.

5. Spencer J. The clinical teaching context: a cause for concern. Med Educ 2003;37:182-3.

6. Langevin S, Hivon R. En quoi l'externat ne s'acquitte $t$-il pas adéquatement de son mandat pédagogique? U. Une étude qualitative fondée sur une analyse systématique de la littérature. Pédagogie Médicale 2007;8:7-23. vision et le modèle de rôle ${ }^{7}$ la pratique du feed-back ${ }^{10}$ ou l'enseignement et l'apprentissage du raisonnement clinique $^{I I}$, ont déjà été publiées dans notre journal.

Ainsi, l'externat et, d'une façon générale, tous les stages en milieu clinique en tant que dispositifs de formation des différents professionnels en sciences de la santé, sont confrontés à plusieurs défis. Des références scientifiques, permettant d'analyser nos programmes et d'élaborer des hypothèses crédibles et raisonnables de solution ou d'amélioration, sont aujourd'hui disponibles. Dès lors, il appartient aux enseignants, aux responsables institutionnels et aux tutelles politiques des différents programmes de formation des professionnels de santé, chacun pour ce qui les concerne, de mettre en ouvre des moyens et des ressources pour développer des projets de formation-action ou de recherche-action pertinents. Dans son rôle de société savante, riche des ressources francophones qu'elle peut mobiliser, la Société internationale francophone d'éducation médicale (SIFEM) pourrait, sur sollicitation, apporter une expertise scientifique et contribuer à valoriser ces travaux.

A cet égard, il serait dommage d'en rester aux déclarations d'intention.

Jean JOUQUAN mailto:jean.jouquan@chu-brest.fr

7. Chamberland M, Hivon R. Les compétences de l'enseignant clinicien et le modèle de rôle en formation clinique. Pédagogie Médicale 2005;6:98-111

8. Tardif J. L'évaluation des compétences. Documenter le parcours de développement. Montréal (QC): Chenelière Education, 2006.

9. Collins A, Brown JS, Newman SE. Cognitive apprenticeship:Teaching the crafts of reading, writing and mathematics. In: Resnick LB (eds). Knowing, learning and instruction: Essays in honor of Robert Glaser.Hillsdale (NJ): Lawrence Erlbaum Associates, 1989:453-94.

10. O'Brien HV, Marks MB, Charlin B. Le feedback (ou rétro-action) : un élément essentiel de l'intervention pédagogique en milieu clinique. Pédagogie Médicale 2003;4:184-91

11. Nendaz M, Charlin B, LeBlanc V, Bordage G. Le raisonnement clinique: données issues de la recherche et implications pour l'enseignement. Pédagogie Médicale 2005;6:235-54. 